\title{
Useful method for evaluation of local irritation using auricular subcutis of rabbits
}

\author{
Kazuhisa Hara¹, Tetsuya Matsuoka², Takuma Hara², Kei Wakabayashi², Mutsumi Kanda², \\ Ryo Ando ${ }^{2}$ and Takumi Yamashita ${ }^{1}$
}

\author{
'Pharmaceutical Research Laboratories, Nippon Kayaku Co., Ltd., 31-12, Shimo 3-chome, Kita-ku, \\ Tokyo 115-8588, Japan \\ ${ }_{2}^{2}$ Kannami Laboratory, Bozo Research Center Inc., 1308-125, Kuwahara-sanbonmatsu, Kannami-cho, Tagata-gun, \\ Shizuoka 419-0101, Japan
}

(Received July 23, 2011; Accepted October 26, 2011)

\begin{abstract}
For the local irritation caused by Doxorubicin hydrochloride (DXR) which have leaked to the subcutis from the vein, the usefulness of the model using the auricular subcutis of rabbits was examined. DXR was administered to the subcutis in the ear auricle, abdominal region and dorsal region, and the local irritation reactions induced were evaluated according to the Draize criteria, by comparison of the damaged area and by the histopathological method. Macroscopic formations of erythema, edema and eschar were observed in the auricular subcutis, but there were no changes in the abdominal or dorsal subcutis. Histopathological examination showed changes such as edema, hemorrhage and necrosis at all administration sites and the changes were most severe in the auricular subcutis among the 3 regions. The reactions in the ear auricle observed were closest to the skin damage noted in humans by administration of DXR. In order to find out why the degree of local damage is different in these 3 regions, Evans blue was administered to these regions to compare its diffusibility in these regions. The diffusibility of Evans blue was lowest in the ear auricle. It is estimated that the difference in the local damage induced by DXR in these regions might be due to the difference in the retention time of DXR in the subcutis. Therefore, the evaluation for local irritation using the auricular subcutis model in rabbits is considered to be useful for estimation of skin damage caused by leakage of DXR to the subcutis.
\end{abstract}

Key words: Local irritation, Doxorubicin hydrochloride, Rabbit, Auricular subcutis

\section{INTRODUCTION}

Chemotherapy is a very useful approach in cancer treatment. However, the anticancer agents available at the present time have severe adverse effects including local damage at the application site (Ignoffo and Friedman, 1980; Rudolph and Larson, 1987) which cause suffering in patients.

For evaluation of the local irritability of drugs, there are various established animal models and appropriate test methods that can be selected depending on the expected clinical route of administration, as follows: eye mucosa irritation study in rabbits (Draize et al., 1944) for ophthalmologic drugs; primary skin irritation study in rabbits (Draize et al., 1944; Ikeda et al., 1970) for transdermal drugs; and vascular irritation evaluation method by retention of the test article in blood vessels (Fukawa et al.,
1975) and intramuscular irritation evaluation methods using vastus lateralis muscle in rabbits (Fukawa et al., 1975; Ochiai et al., 1985) for injection drugs. For evaluation of irritation reactions induced by subcutaneous drugs or by the leakage of injections to subcutis, evaluation is done by using the dorsal subcutis (Morita et al., 1994; Tachita et al., 1992), abdominal subcutis (Nakai et al., 1990; Nakamura et al., 1992) and the auricular subcutis (Uchida et al., 2001) in rabbits or alternatively by conducting an intramuscular irritation study in rabbits, but there is no established evaluation method. Among various anticancer agents, vesicant drugs as typified by Doxorubicin hydrochloride (DXR) are known to induce severe local inflammation, even if only a small amount leaks out of the blood vessel, and then leave irreversible damage (Harwood and Aisner, 1984; Rudolph and Larson, 1987). In order to avoid such damage, evaluation using an appro-

Correspondence: Kazuhisa Hara (E-mail: kazuhisa.hara@nipponkayaku.co.jp) 
priate model for subcutaneous local irritation is necessary before administration to humans. The authors examined an irritation evaluation model, which is highly extrapolative to humans, by comparing the local damage caused by subcutaneous administration of DXR to the subcutis in the dorsal, abdominal and auricular rabbit skin.

\section{MATERIALS AND METHODS}

\section{Test article}

DXR (content 99.54\%) was received from Mercian Corporation (Tokyo, Japan). Evans blue was purchased from Wako Pure Chemical Industries, Ltd. (Tokyo, Japan). DXR was dissolved in 5\% glucose solution for injection (Otsuka Pharmaceutical Factory, Inc., Tokushima, Japan) at $2 \mathrm{mg} / \mathrm{ml}$. Extravasation of highly concentrated solutions of DXR produce a greater incidence of reactions than would the appropriately diluted drug (Bowers and Lynch, 1978). This concentration which is generally the highest concentration solution used for intravenous bolus injection in humans is considered to be sufficient to cause severe local damage at the administration site. Evans blue was dissolved in water for injection (Otsuka Pharmaceutical Factory, Inc.) at $5 \mathrm{mg} / \mathrm{ml}$. Evans blue was selected so that the measurement of the diffusion area would be easier.

\section{Test system and animal husbandry}

Female Japanese White rabbits at 16-19 weeks of age were purchased from Japan Laboratory Animals, Inc. (Tokyo, Japan) and used in this study. They were housed individually in aluminum cages (W360 mm $\times$ D550 mm $\times$ H350 mm: Nihon Cage Co., Ltd., Tokyo, Japan) which were placed in an animal room where the temperature was kept at $23 \pm 5^{\circ} \mathrm{C}$, the relative humidity at $55 \pm 25 \%$, air ventilation at 11-14 times per hour and lighting for $12 \mathrm{hr}$ a day (07:00 to 19:00), and acclimated to the study environment. They were allowed free access to pelleted diet RC4 (Oriental Yeast Co., Ltd., Tokyo, Japan) and tap water (Fujimi Water Union, Shizuoka, Japan) using the automatic water supply system.

The animal care and experimental procedures were performed in accordance with the animal welfare guidelines of Bozo Research Center Inc. (Shizuoka, Japan) and carried out in accordance with the standards of the Institute of Laboratory Animals Resources Guide.

\section{Comparison of local irritation reactions caused by subcutaneous administration of DXR to the auricular, dorsal and abdominal regions}

Animals weighing 2.18-3.39 kg were used. Each group consisted of 9 animals and a total of 3 groups the auricular subcutaneous administration group, the dorsal subcutaneous administration group and the abdominal subcutaneous administration group - were provided. The administration site was approximately midway between the central auricle and the base of the ear between the intermediate branch of the anterior auricular vein and the

Table 1. Draize Criteria

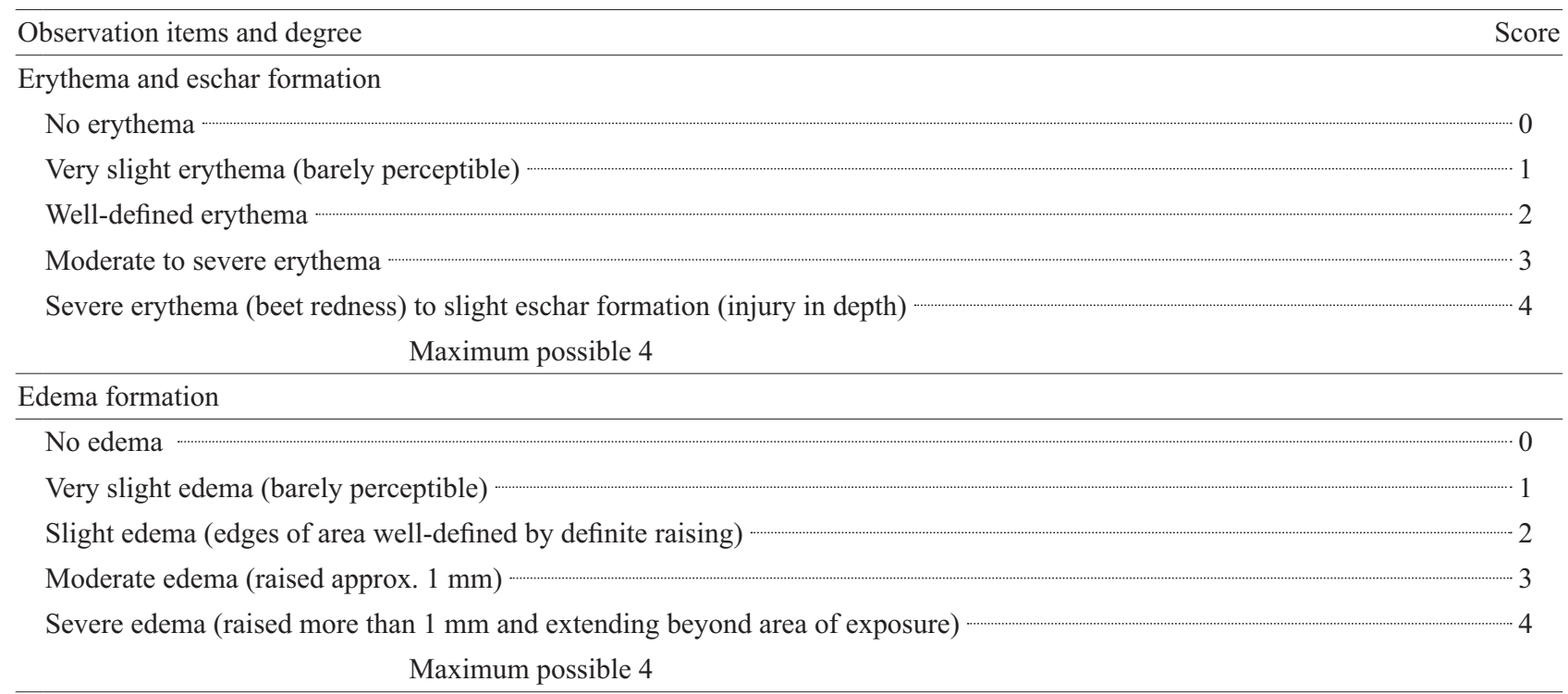


posterior auricular vein for the ear auricle, in the center of the right and left scapula for the dorsal region, and in the center but approximately $2 \mathrm{~cm}$ lower than the final rib for the abdominal region. The fur of each administration region was clipped using an electric clipper before administration, and then the animal was dressed with an Elizabeth collar. $0.1 \mathrm{ml}$ of the DXR solution was administered subcutaneously each to the auricular, dorsal and abdominal regions using 1-ml disposable syringe (Terumo Corporation, Tokyo, Japan) with $27 \mathrm{G}$ injection needle (Terumo Corporation). After administration, the injection needle was removed and the administration site was marked with oil-based ink. The administration site was observed for 28 days after administration. Every day from the day of administration (Day 0) to day 7 after administration (Day 7) and on Days 10, 14, 17, 21, 24 and 28, dermal reactions (erythema, edema and eschar formation) observed were classified according to the Draize criteria (Table 1). At the same time, the major and minor axes were measured and the area of damage was calculated $(\pi$ $\times$ Major axis $/ 2 \times$ Minor axis/2). In addition, in order to evaluate organic lesions, pathological examination was done as follows: on Days 5, 10 and 28, 3 animals per group were euthanized by administration of pentobarbital sodium (Tokyo Kasei Kogyo Co., Ltd., Tokyo, Japan) solution (in-house preparation) via the posterior auricular vein at the ear base, portions of the skin in the auricular, dorsal and abdominal regions were collected, fixed in phosphate buffered $10 \%$ formalin, axial sections including the injection site and axial sections in the center of the administration site were trimmed, embedded in paraffin, hematoxylin/eosin (H\&E) stained specimens were prepared, and observed using a light microscope. For the scores of macroscopic skin reactions and the area of skin damage, mean with standard deviation was calculated for each administration site. The measured values and the results of histopathological examination were compared comprehensively among the administration sites.

\section{Comparison of the diffusibility of pigments at the subcutaneous site of auricular, dorsal and abdominal regions}

Animals weighing 2.22-4.10 kg were used. Each group consisted of 9 animals and a total of 3 groups - the auricular subcutaneous administration group, the dorsal subcutaneous administration group and the abdominal subcutaneous administration group - were provided. The site and method of administration were the same as for the study for comparison of irritation by DXR. After administration of Evans blue, the injection needle was removed, the injection site was marked using an oil-based ink, and
3 animals were euthanized by administration of pentobarbital sodium (Tokyo Kasei Kogyo Co., Ltd.) solution (in-house preparation) via the posterior auricular vein at the ear base immediately after, or 1, 2 or $4 \mathrm{hr}$ after administration. For the auricle, the major and minor axes of the area showing blue-colored subcutis were measured. For the dorsal and abdominal regions, a portion of the skin including the injection site in the center was removed and the major and minor axes of the area showing blue-colored subcutis were measured in the same manner. The area was calculated from the measured major and minor axes $(\pi \times$ Major axis/ $2 \times$ Minor axis/2).

\section{RESULTS}

\section{Skin damage in the auricular, dorsal and abdominal regions after administration of DXR}

In the auricular region, skin damage was observed as erythema and edema from the day following administration. In the dorsal region and abdominal region, there were no dermal changes during the observation period.

Mean scores of these dermal irritation changes according to the Draize standard are shown in Fig. 1 and the photographs in Figs. 2-1, 2-2 and 2-3. The skin damage in the auricular region was severe from the day following administration (Day 1) to Day 3, the damage remained severe from Day 3 to Day 6 when erythema was score 2 or 3 and edema score 1 or 2 , and erythema was increased to score 4 accompanied by eschar formation on Day 7. From Day 10 to Day 14, erythema with eschar persisted at score 4 while edema was decreased to score 1 or 2 or no longer observed. From Day 14, eschar was remaining and erythema was of score 4 , but edema was no longer observed.

\section{Region of skin damage at each administration site after administration of DXR}

The results of measurement of the areas with skin irritation damage are shown in Fig. 3. Measurement of skin damage was possible only in the auricle and there were no dermal changes in the dorsal or abdominal region.

The area with skin damage in the auricle spread abruptly on Day 1, reached the maximum $\left(779 \mathrm{~mm}^{2}\right)$ on Day 2 and persisted without any remarkable variations until Day 6 . The area reduced abruptly $\left(194 \mathrm{~mm}^{2}\right)$ from Day 7 to Day 14, slowly from Day 14 thereafter and became $59 \mathrm{~mm}^{2}$ on Day 28.

\section{Histopathological examination of the subcutis in the auricular, dorsal and abdominal regions after administration of DXR}

The results of histopathological examination are shown 


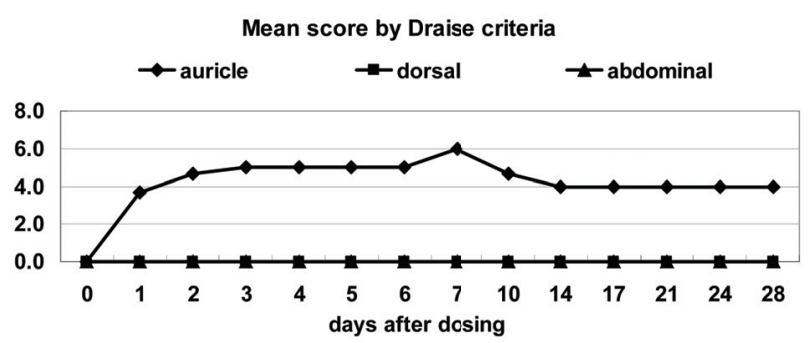

Fig. 1. Mean score of macroscopic skin irritation reactions in the auricular, dorsal and abdominal regions by the Draize Standard.

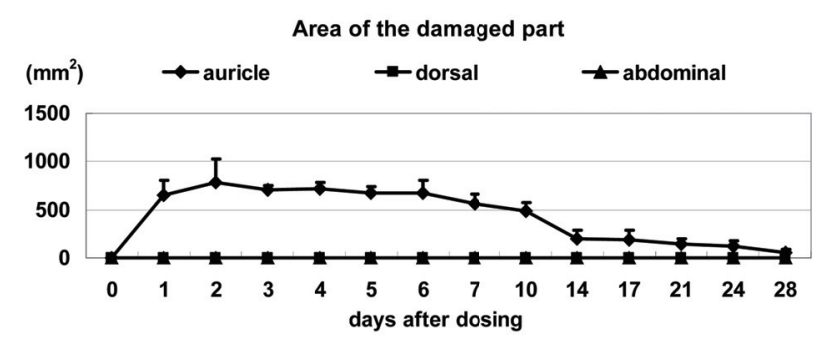

Fig. 3. Area of the part damaged by skin irritation in the auricular, dorsal and abdominal regions.

in Table 2. In the auricle, changes were observed in the epidermis and subcutis: eschar formation and erosion/ ulcer in the epidermis and edema, hemorrhage, necrosis and cell infiltration in the subcutis on Day 5 (Figs. 4-1, 4-2, 4-3); hyperplasia in addition to eschar formation and erosion/ulcer in the epidermis and granulation tissue/ fibrosis in addition to edema, hemorrhage, necrosis and cell infiltration in the subcutis on Day 10; and hyperkeratosis in addition to eschar formation and hyperplasia in the epidermis and necrosis, granulation tissue/fibrosis and cell infiltration in the subcutis on Day 28.

In the abdominal region, changes were observed in the subcutis but not in the epidermis: edema, hemorrhage, necrosis and cell infiltration in the subcutis on Day 5; granulation tissue/fibrosis in addition to edema, hemorrhage, necrosis and cell infiltration in the subcutis on Day 10; and granulation tissue/fibrosis and cell infiltration in the subcutis on Day 28. The tissue damage in the abdominal subcutis was largely the same as for the tissue damage in the auricular subcutis, but was less severe.

In the dorsal region, changes were observed in the epidermis and subcutis: cell infiltration in the subcutis on Day 5; hyperplasia in the epidermis and necrosis, granulation tissue/fibrosis and cell infiltration in the subcutis on Day 10; and hyperplasia in the epidermis and necrosis

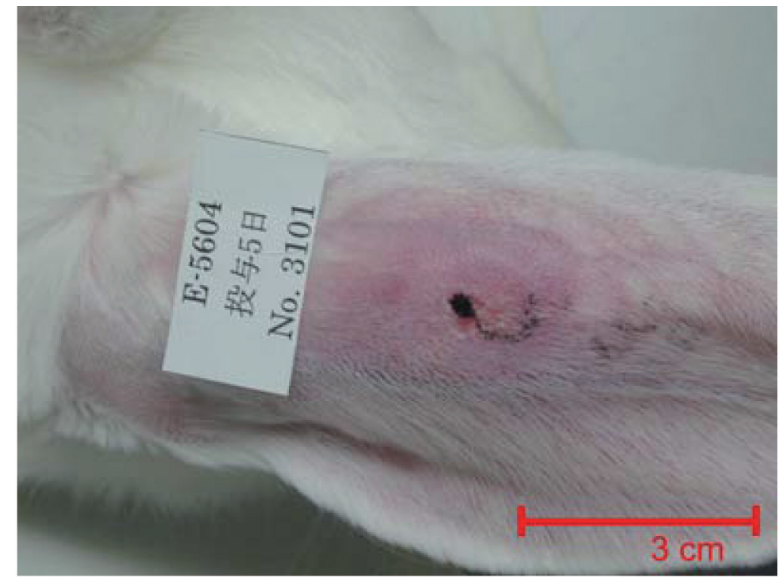

Fig. 2-1. Auricular subcutis, Day 5 after administration of DXR. Score of Draize standard: Erythema 3, Edema 2.

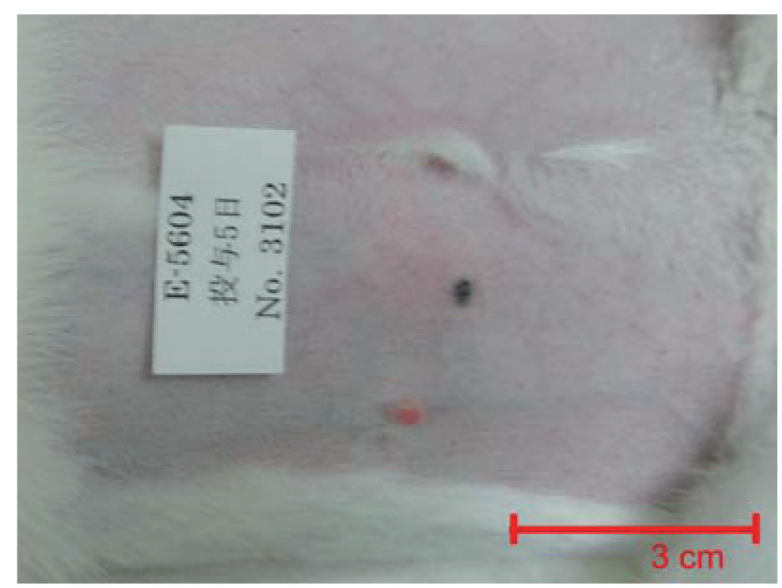

Fig. 2-2. Abdominal subcutis, Day 5 after administration of DXR. Score of Draize standard: Erythema 0, Edema 0.

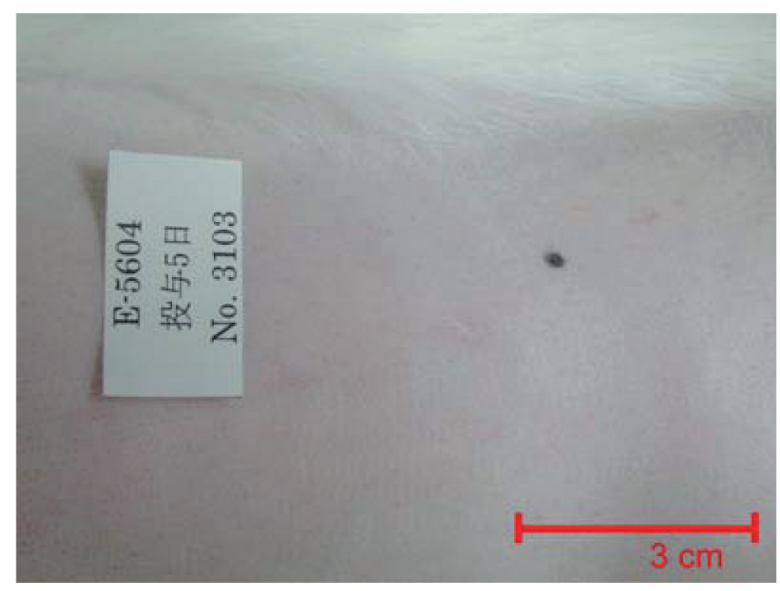

Fig. 2-3. Dorsal subcutis, Day 5 after administration of DXR. Score of Draize standard: Erythema 0, Edema 0. 
Local irritation using auricular subcutis of rabbits

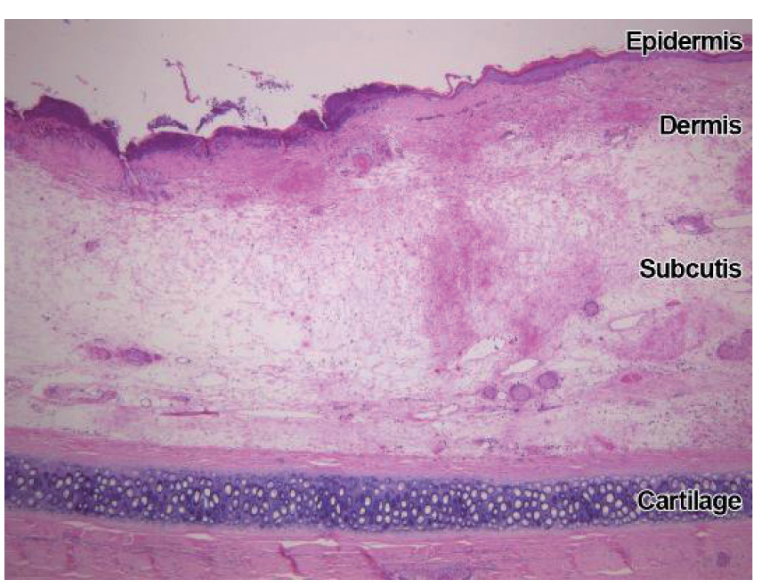

Fig. 4-1. Auricular subcutis, Day 5 after administration of DXR. Edema, hemorrhage and necrosis in dermis to subcutis and erosion/ulcer in epidermis were observed.

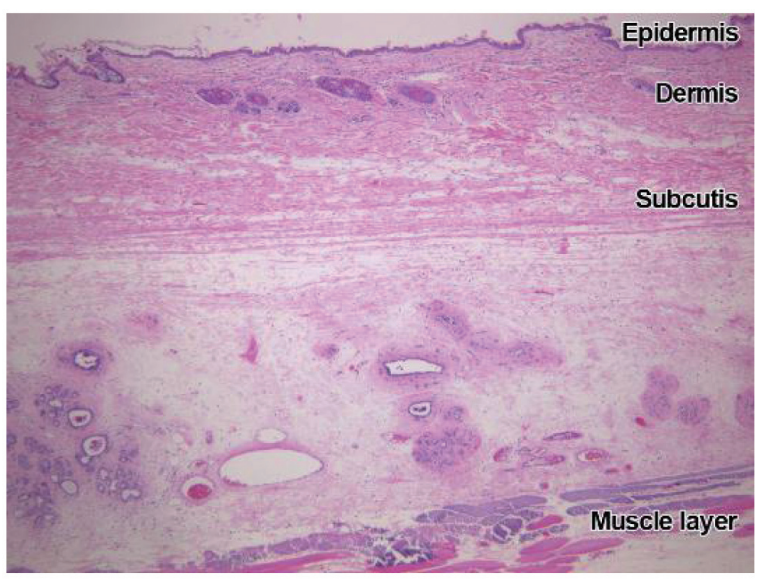

Fig. 4-2. Abdominal subcutis, Day 5 after administration of DXR. Edema and necrosis in dermis to subcutis whereas no remarkable changes in epidermis were observed.

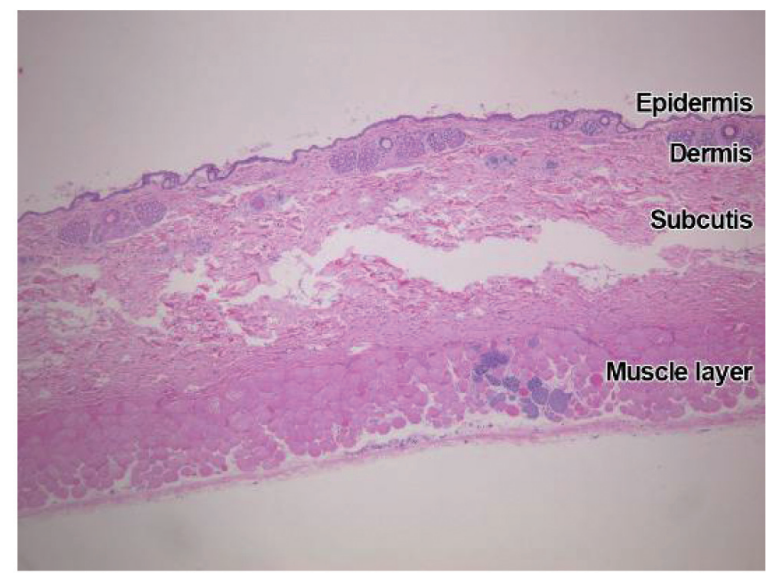

Fig. 4-3. Dorsal subcutis, Day 5 after administration of DXR. No remarkable changes in epidermis, dermis and subcutaneous tissue.

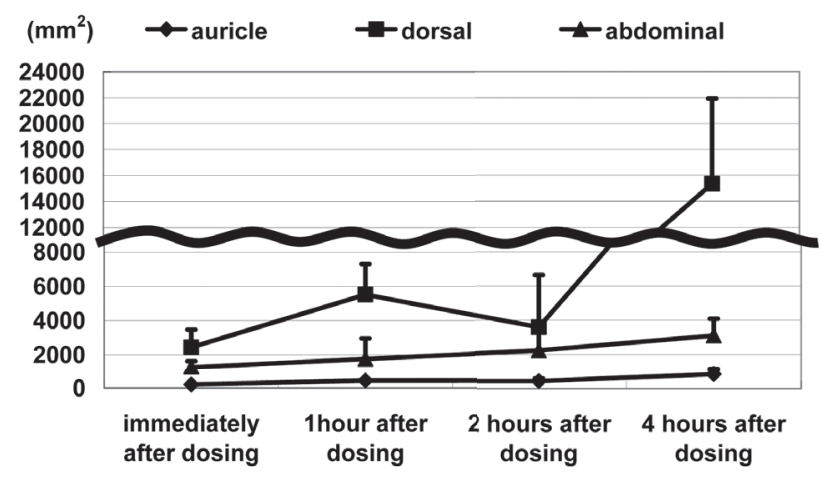

Fig. 5. Time course of the diffusion area of Evans blue in subcutis in auricular, dorsal and abdominal regions.

in the subcutis on Day 28. These changes were observed in 1 or 2 animals and the degree of change was comparatively slight and thus weakest among the 3 regions.

\section{Time course of the diffusion area of Evans blue in the subcutis in the auricular, dorsal and abdominal regions}

The time course of the diffusion area of Evans blue is shown in Fig. 5 and the photographs in Figs. 6-1, 6-2, 6-3. The diffusion area of Evans blue was smallest in the auricle and larger in the abdominal region and dorsal region in this order.

Compared to the area of diffusion in the auricular subcutis, the diffusion areas in the abdominal and dorsal regions were respectively 6.0 and 11.6 times that of the auricular region immediately after administration, 4.0 and 12.5 times at $1 \mathrm{hr}$ after administration, 4.9 and 8.0 times at $2 \mathrm{hr}$ after administration and 3.6 and 18.0 times at $4 \mathrm{hr}$ after administration.

\section{DISCUSSION}

Local irritation by DXR was compared using the subcutis in the auricular, abdominal and dorsal regions in the rabbit. Macroscopically observable irritation reactions were observed in the auricle, but such reactions were not observed in the dorsal or abdominal regions. Local damage in the auricular subcutis reached the peak several days after administration, largely persisted until approximately 1 week after administration, then decreased quickly until week 2 after administration, and decreased slowly thereafter. The irritation reactions observed were compared using two indices: macroscopic assessment using the Draize criteria and the area of the damaged region. In the macroscopic assessment, since the judging criteria 
K. Hara et al.

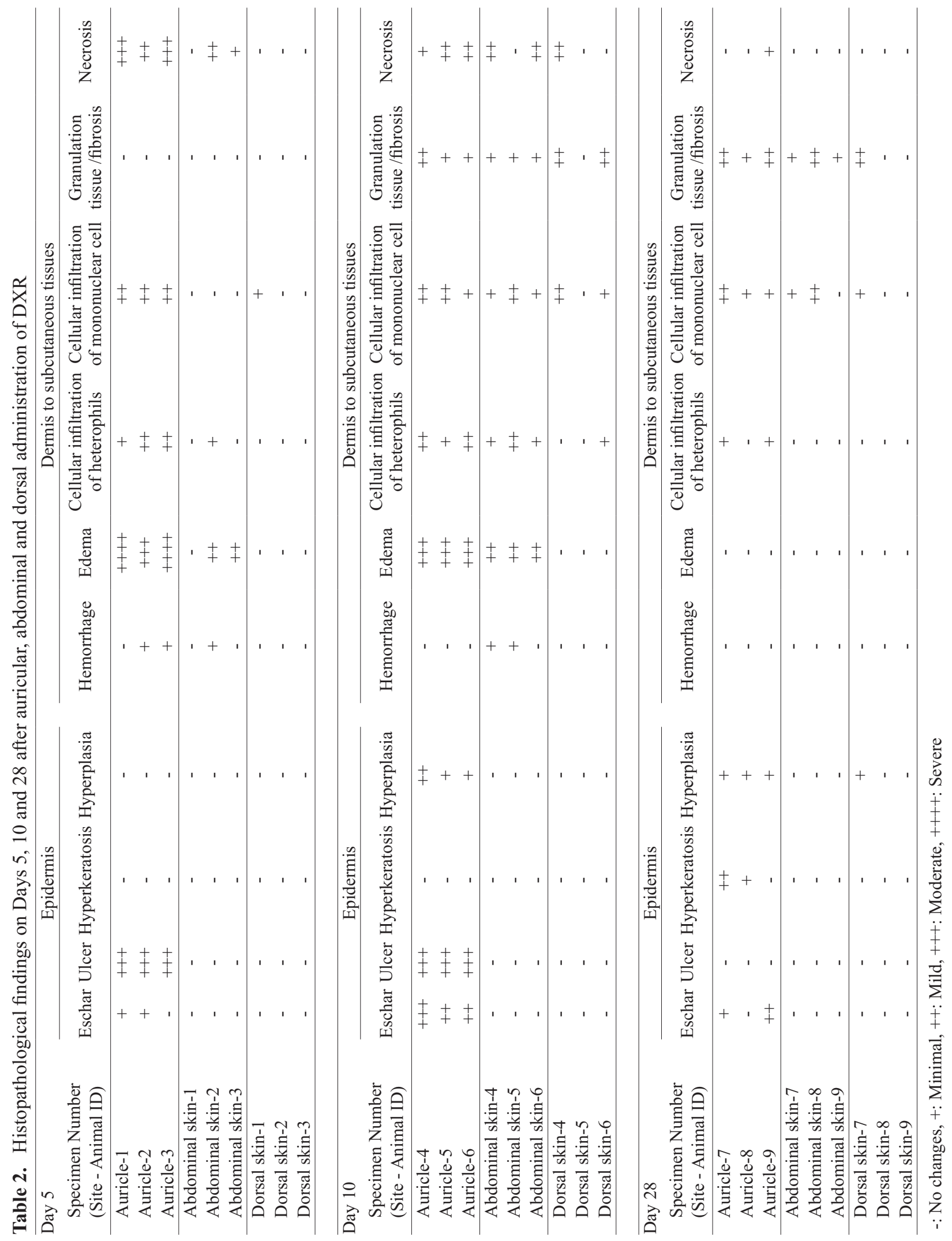

Vol. 37 No. 1 
Local irritation using auricular subcutis of rabbits

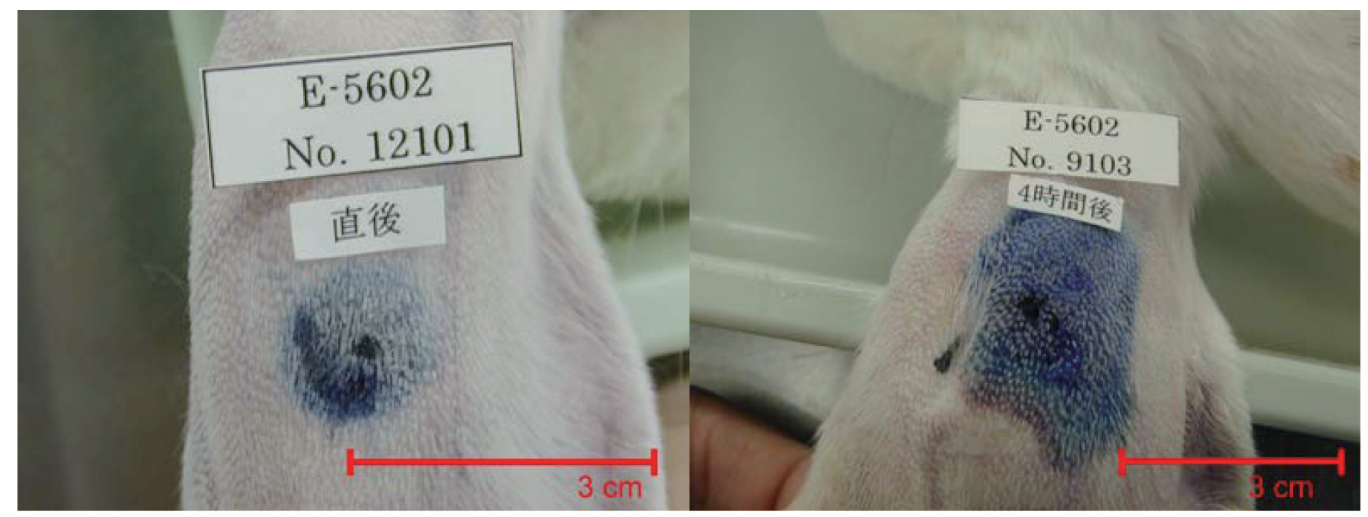

Fig. 6-1. Auricular subcutis, immediately (left) and $4 \mathrm{hr}$ (right) after administration of Evans blue. Size of the time indication label: $1 \mathrm{~cm} \times 3 \mathrm{~cm}$.

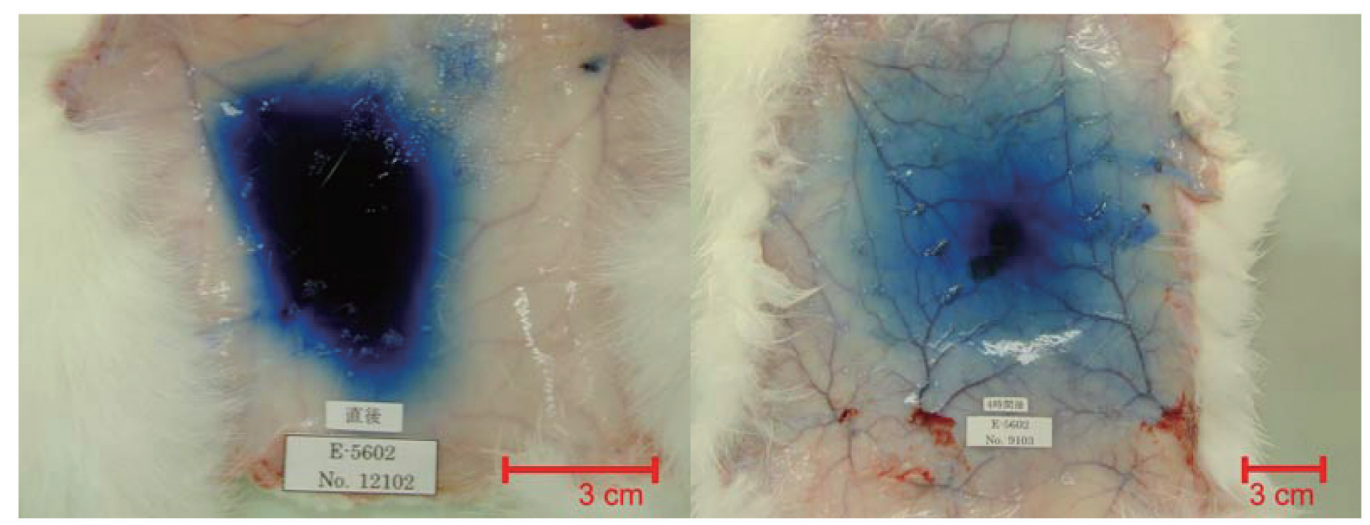

Fig. 6-2. Dorsal subcutis, immediately (left) and $4 \mathrm{hr}$ (right) after administration of Evans blue. Size of the time indication label: $1 \mathrm{~cm} \times 3 \mathrm{~cm}$.

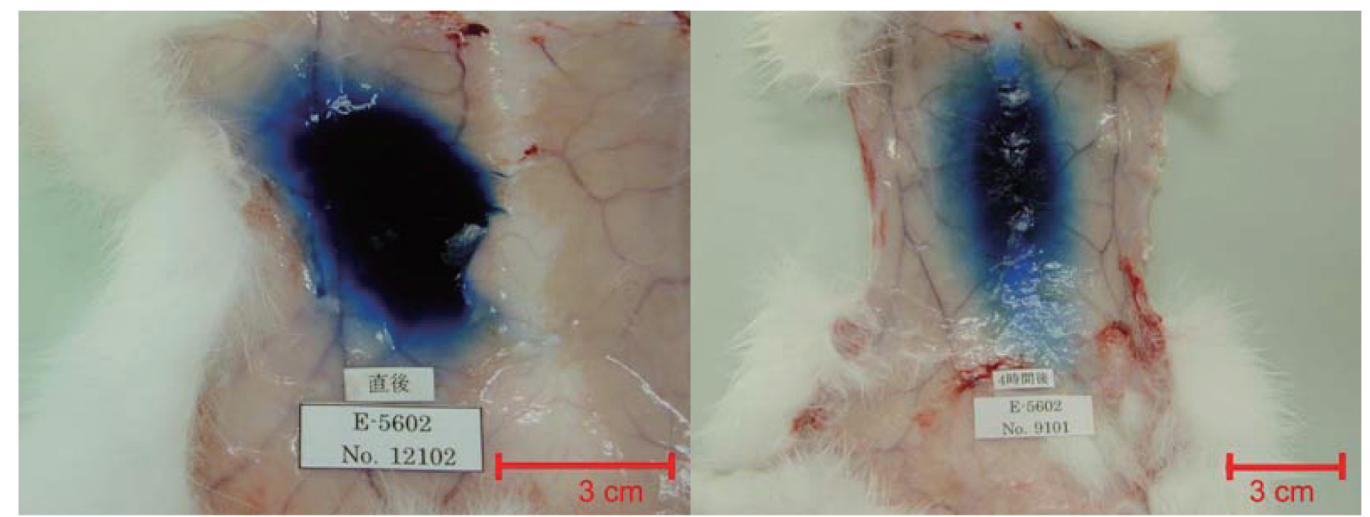

Fig. 6-3. Abdominal subcutis, immediately (left) and $4 \mathrm{hr}$ (right) after administration of Evans blue. Size of the time indication label: $1 \mathrm{~cm} \times 3 \mathrm{~cm}$.

where eschar formation increases the score to the maximum is used, the presence of eschar formation might not decrease the score even if the area of damage were getting smaller with the passage of time, and thus the proc- ess of recovery from Day 7 onward might not be reflected in the score. In contrast, the area of the damaged region which directly reflects the process of recovery could be used as a useful indicator showing the process of recov- 
ery from the local damage. If treatment-related skin damage is observed in humans, not only the severity of damage but also its reversibility is an important point. Thus, it was thought to be very important to measure the damaged area which might represent the process of skin damage from its emergence to regression. In the histopathological examination of the administration sites, changes were most severe in the auricle also noted at macroscopic observation. In the auricle, severe tissue damage from the subcutis to the epidermis was observed. In the abdominal region, changes observed in the subcutis were comparable to those in the auricle but there were almost no changes in the dermis or epidermis. In the dorsal region, changes were weakest of the 3 regions; only remarkably weak tissue damage was observed and there was no damage in the tissue of some animals. If certain anticancer agents that are classified as vesicant drugs including DXR leak outside the vein in humans, erythema and swelling would occur at first, bulla formation, induration and necrosis would follow with the progress in local inflammation, and ulcer could be formed (Ishihara and Yamazaki, 1992; Yanagawa et al., 1992). In the local damage that occurred in the auricular, abdominal and dorsal subcutis by administration of DXR, only the damage in the auricular subcutis was similar to that of humans, forming ulcer in the same manner. Tone et al. reported that subcutaneous administration of DXR at $5 \mathrm{mg} / \mathrm{ml}$ (dose volume: 0.1 $\mathrm{ml}$ ) to the dorsal region of rabbits caused no macroscopic changes (Tone et al., 1986) while Uchida et al. (2001) reported that subcutaneous administration of DXR at 200 and $450 \mu \mathrm{g} / \mathrm{ml}$ (dose volume: $0.5 \mathrm{ml}$ ) to the auricular region of rabbits caused erythema, swelling and eschar formation. The results of these experiments agree with those of our experiment indicating that the auricular subcutis is more susceptible to local irritation by DXR than the dorsal subcutis. As described above, it is estimated that the auricular subcutis of the rabbit is the region which would best reflect the irritation of DXR leaked around the vein at the time of intravenous administration in humans. On the other hand, irritability of DXR in the abdominal and dorsal subcutis was weaker than the irritation that occurs in humans and thus estimation of skin damage in humans using these regions might be incorrect.

In order to investigate why the local damage by DXR was different in the subcutis in the auricular, abdominal and dorsal regions in rabbits, diffusibility of a test article in these regions was examined. Evans blue was useful for predicting the spread of chemicals under the skin. Evans blue was administered to 3 regions and the diffusion area was measured serially. In the results, the area of diffusion was the largest in the dorsal subcutis, and became small- er in the abdominal subcutis and then the auricular subcutis. Compared to the area of diffusion in the auricular subcutis, it was approximately 8 to 18 -fold in the dorsal region and approximately 4 to 6 -fold in the abdominal region, and thus the rate of diffusion was quickest in the dorsal subcutis. The difference in the rate of diffusion might be caused by the difference in the tissue structure of these regions. In the skin of the auricle, connective tissues under the dermis are tightly bound and elasticity of the skin is small. On the other hand, the skin in the abdominal and dorsal regions, which are rich in fatty tissues in the subcutaneous connective tissues, is largely elastic and the binding between the subcutaneous tissues and the muscle layer is loose. Due to such differences, it is estimated that a substance which is administered to the auricular subcutis tends to stay while the substance administered to the abdominal or dorsal subcutis might quickly diffuse between the subcutaneous tissues and muscle layer. Comparison of the difference in diffusibility of Evans blue in subcutaneous regions and the degree of local damage by DXR revealed that the substance with smaller diffusibility caused more severe local damage. Therefore, it was estimated that the reason for the difference in the local damage at the administration site by administration of DXR could be the difference in its retention time after subcutaneous administration due to the difference in the structure of the tissue.

Based on the above, it is expected that the local irritation evaluation method using the auricular subcutis model in rabbits is useful for estimation of skin damage by DXR which occur in humans. In addition, it is considered that the method is also applicable to estimate local irritation by anticancer drugs or other general pharmaceuticals at the time of leakage out of the blood vessel or to evaluate local irritation by the subcutaneous drugs.

\section{ACKNOWLEDGMENTS}

The authors would like to thank Mr. Pete Aughton, D.A.B.T., Dip. R. C. Path., ITR Laboratories Canada Inc., for proofreading.

\section{REFERENCES}

Bowers, D.G.Jr. and Lynch, J. B. (1978): Adriamycin extravasation. Plast. Reconstr. Surg, 61, 86-92.

Draize, J.H., Woodard, G. and Calvery, H.O. (1944): Methods for the study of irritation and toxicity of substances applied topically to the skin and mucous membranes. J. Pharmacol. Exp. Ther., 82, 377-390.

Fukawa, K., Ito, Y., Misaki, N. and Bando, K. (1975): A new method for the local irritation test. I. Tissue regeneration test for intra- 
Local irritation using auricular subcutis of rabbits

muscular acetic acid injection. Yakugaku Zasshi, 95, 1307-1316.

Fukawa, K., Ito, Y., Misaki, N. and Nomura, S. (1975): Thrombophlebitis following intravenous injection--an experimental study. Folia Pharmacologica Japonica, 71, 307-315.

Harwood, K.V. and Aisner, J. (1984): Treatment of chemotherapy extravasation: current status. Cancer Treat. Rep., 68, 939-945.

Ignoffo, R.J. and Friedman, M.A. (1980): Therapy of local toxicities caused by extravasation of cancer chemotherapeutic drugs. Cancer Treat. Rev., 7, 17-27.

Ikeda, Y., Horiuchi, S., Yoshimoto, H., Furuya, T., Kawamata, K. and Kaneko, T. (1970): Investigation of dermal irritation test methods. Iyakuhin Kenkyu, 1, 23-27.

Ishihara, K. and Yamazaki, N. (1992): Extravasation of anticancer drug and its management. Japanese Skin Cancer Society. Skin Cancer, 7, 117-128.

Morita, H., Shimomura, K. and Sunaga, M. (1994): Local subcutaneous irritation study of OCT-43 in rabbits. Jpn. Pharmacol. Ther., 22, 181-189.

Nakai, Y., Nakamura H., Chatani, F. and Sasaki, S. (1990): Subcutaneous irritation study of TAP-144-SR in rabbits. Jpn. Pharmacol. Ther., 18, 673-680.

Nakamura, H., Chatani, F., Oshima, Y. and Nakai, Y. (1992): Local irritation study of TGP-943 in rabbits. Kiso To Rinsho, 26, $3107-$
3117.

Ochiai, T., Matsumoto, K., Sekita, K., Uchida, O., Kawasaki, Y. and Furuya, T. (1985): Local irritation of isotonic acetic acid solutions of the rabbit $\mathrm{M}$. vastus lateralis and confirmation of the injected sites. Jikken dobutsu, 34, 399-406.

Rudolph, R. and Larson, D.L. (1987): Etiology and treatment of chemotherapeutic agent extravasation injuries: A review. J. Clin. Oncol., 5, 1116-1126.

Tachita, T., Yamamae, H., Iwatate, K. and Nakamura, T. (1992): A locally-stimulative test in rabbits of hydrochloric acid idarubicin. J. New Rem. Clin., 41, 3014-3032.

Tone, H., Kiyosaki T., Cuthbert, J.A., Carr, S.M.A. and Aitken, R. (1986): Acute local irritative effect of (2'R)-4'-O-tetrahydropyranyladriamycin, a new antitumor antibiotic. Jpn. J. Antibiot., 39, 569-581.

Uchida, K., Kaneko, K., Nagata, T., Kado, S., Mizutani, T., Shimizu, S., Iwata, S., Sasaki, T. and Onoue, M. (2001): Local irritation studies of CAMPTO ${ }^{\circledR}$ inj. (CPT-11) in rabbits. Jpn. Pharmacol. Ther., 29, 135-149.

Yanagawa, S., Osumi, M., Kumai, R. and Ishihara, K. (1992): The management of anticancer-Drug extravasation. Rinsho hifuka, 46, 169-174. 